\title{
Alles Enzephalitozoonose - oder gibt es da noch mehr?
}

\author{
Jutta Hein
}

Immer wenn ein Kaninchen mit neurologischen Störungen wie Vestibularsyndrom oder Lähmungen, Augenveränderungen oder Niereninsuffizienz in der Praxis vorgestellt wird, ist man auch ohne Diagnostik oft schnell mit der Diagnose „Enzephalitozoonose“ bei der Hand. Aber ist die Enzephalitozoonose tatsächlich so häufig? Wie erfolgt die Diagnose? Welche Differenzialdiagnosen kommen infrage? Wie sollte die Therapie aussehen und wie ist die die Prognose?

\section{Enzephalitozoonose - was ist das?}

Die Enzephalitozoonose ist eine durch Encephalitozoon cuniculi hervorgerufene, weltweit verbreitete Krankheit der Säugetiere (inkl. Mensch), Vögel, Fische und Arthropoden. Klinische Symptome treten aber zumeist nur bei Kaninchen, Mäusen, Ratten und Rhesusaffen auf [20].

E.cuniculi wurde erstmals 1922 beschrieben [28] und morphologisch der Ordnung der Microsporida zugeordnet. Aufgrund biochemischer und molekularer Merkmale sind diese Parasiten jedoch eher mit den Pilzen als den Protozoen verwandt [15].

Die Infektion erfolgt über Sporen, die vor allem mit dem Urin infizierter Tiere ausgeschieden werden und in der Außenwelt bis zu 2 Jahren überleben. Die Sporen werden bei Futteraufnahme und Fellpflege in erster Linie oral (aber auch diaplazentar, durch Arthropoden etc.) aufgenommen und infizieren zunächst die Darmzellen. Innerhalb der Zellen erfolgt die asexuelle Vermehrung (Sporogonie), die zur Zerstörung der infizierten Zelle führt. Die Zellschädigung erfolgt direkt (Platzen der Zelle) und indirekt (überschießende zelluläre Immunreaktionen).
Die freiwerdenden Sporen gelangen dann mit dem Blutstrom in den gesamten Körper, v.a. aber in besonders gut durchblutete Organe, wie:

- Lunge

- Leber

- Nieren

- ZNS (ab der 9. Woche p.i., [3])

Die Folgen sind Zelluntergang, multifokale, granulomatöse Entzündungen mit Bildung narbiger Einziehungen in Organen und Pseudozystenbildung mit entsprechenden Symptomen [2], [7].

\section{Symptomatik}

Die Ausbildung klinischer Symptome ist abhängig vom Ausmaß der Schädigung in den betroffenen Organen und von der Immunkompetenz des Wirtes.

\section{.konkret}

Immunkompetente Tiere halten die Infektion oft ein Leben lang unter Kontrolle.

Bei diesen Tieren bleibt die Zellschädigung gering und wird nicht klinisch manifest [10]. Kommt es zur Immunsuppression (z.B. durch Krankheit, Stress, Glukokortikoidgabe etc.), können sich die Erreger ungehindert vermehren und Folgeschäden werden klinisch manifest. Welche Symptome auftreten hängt dann davon $\mathrm{ab}$, welche Organe zu diesem Zeitpunkt am meisten geschädigt werden. Im Gegensatz zu einem Befall in anderen Organen (Leber, Lunge etc.) können bereits bei kleinen zerstörten Gehirnarealen massive klinische Symptome auftreten [2].
Die häufigsten Symptome betreffen:

- ZNS

- Ataxie

- Parese (oberes motorisches Neuron)

- Kopfschiefhaltung

- Kreislaufen

- Rollen

- Nystagmus

- Muskelkontraktionen

- Blasenschwäche

- Nieren (interstitielle Nephritis)

- Azotämie

- Polyurie

- Augen

- Katarakt

- Netzhautveränderungen

- Uveitis

Andere Schäden (z. B. Myokarditis, Hepatitis, Splenitis, Pneumonie, Aborte) fallen eher seltener auf [10], [26]. Nach einer aktuellen deutschen Studie [8] hatten 90\% (82/91) der Kaninchen mit bewiesener Enzephalitozoonose (Nachweis durch PCR und/oder Sektion) ZNS-Probleme (Vestibularsyndrom: 71\%, Paresen: 19\%); 29\% (26/91) Nieren-Probleme (Azotämie: 18\%, PD/PU: 12\%) und 12\% (11/91) Augen-Probleme. Die Zahlen decken sich weitgehend mit denen in der aktuelleren Literatur [10], [14].

\section{Diagnostik}

\section{Wann sollte man auf E. cuniculi testen?}

Getestet werden sollte immer,

- wenn die Kaninchen „typische Symptome“ (z.B. neurologische Störungen, Augenveränderungen oder eine Niereninsuffizienz) zeigen, insbesondere wenn in den Wochen vor dem Auftreten der Symptome Grund für eine mögliche Immunsuppression gegeben war (z.B. Stress, Umzug, neues Partnertier) 
- wenn die Kaninchen wiederholt Infektionen (z.B. Schnupfen, Syphilis, Zystitis) haben und/oder chronisch krank erscheinen - E.cuniculi supprimiert die humorale und zellmediierte Immununität [24], sodass infizierte Tiere anfälliger für andere Krankheiten sind

- wenn ein neues Kaninchen in den Bestand aufgenommen werden soll, unabhängig von den Symptomen

\section{Nachweisverfahren}

Grundsätzlich sollte man zwischen „E.cuniculi-infizierte“ und „klinisch manifest erkrankte“ Patienten unterscheiden. Zum Nachweis der E.-cuniculi-Infektion gibt es verschieden Verfahren.

\section{Histopathologische Untersuchung}

Sie gilt als Goldstandard für die Diagnose der Enzephalitozoonose post mortem. Für die Praxis ist sie nur von Bedeutung, wenn ein Tier vor Diagnosestellung verstirbt zum Schutz der Partnertiere.

\section{Direkter Erregernachweis}

Der direkter Erregernachweis kann erfolgen mittels:

- Trichromfärbung im Urin (89\% falschnegativ [12])

- Urin-PCR (60\% falsch-negativ [8], [12])

- Liquor-PCR (89\% falsch-negativ [12])

Der Sporennachweis aus dem Kot ist nicht erfolgreich [4].

Nur ein positiver Sporennachweis (PCR, Trichromfärbung) ist beweisend für eine Infektion, bedeutet jedoch nicht zwangsläufig, dass die Infektion auch Ursache der Symptome ist. Auch infizierte Tiere, die klinisch inapparent sind, scheiden intermittierend Sporen aus.

\section{.konkret}

Ein negativer Sporennachweis schließt eine Infektion nicht aus.

\section{Indirekter Erregernachweis}

Der indirekte Erregernachweis erfolgt als Antikörpertiterbestimmung (meist IgG) mittels IFT oder ELISA. Die Antikörper sind ca. 14-21 Tage nach oraler Infektion im Blut nachweisbar [27].
Die Aussagekraft des Antikörpernachweises war lange Zeit umstritten. Grund hierfür war die Annahme, dass ohnehin bis zu 95\% der Tiere mit E.cuniculi infiziert wären. Diese Aussage beruhte jedoch auf einer Untersuchung in geschlossenen Laborbeständen in England [9]. Antikörperprävalenzen bei Heimtierkaninchen sind weltweit unterschiedlich (20$60 \%)$, Wildkaninchen sind nur selten infiziert. In Deutschland werden die Prävalenzen mit 41-45\% angegeben [6], [16], [17]. In Bayern [11] waren insgesamt nur 39\% (302/773) der untersuchten Kaninchen E-cuniculi-Antikörper-positiv, wobei sich die Prävalenzen wie folgt aufteilten:

- $18 \%$ (39/218) bei klinisch gesunden Kaninchen

- $14 \%(31 / 219)$ bei kranken Tieren mit anderen Symptomen

- $60 \%$ bei Tieren mit Verdacht auf eine Infektion

- 96\% (91/95) bei Kaninchen mit bewiesener Infektion (PCR oder Sektion)

Es sind also deutlich weniger Kaninchen infiziert als angenommen und die Sensitivität des E.-cuniculi-IgG-Antikörpertests ist mit $96 \%$ sehr gut [11].

Ein positiver Antikörpernachweis ist beweisend für eine bestehende, lebenslang persistierende Infektion, sagt aber nicht aus, ob die Infektion auch Ursache der Symptomatik ist.

Auch die Höhe des Antikörpertiters ist hierfür nicht von Bedeutung [26], da die meisten infizierten Tiere lebenslang hohe Titer aufweisen. Maternale Antikörper können bis zur 8. Lebenswoche nachgewiesen werden [19].

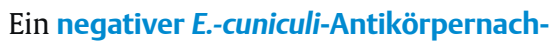
weis schließt eine Infektion mit hoher Wahrscheinlichkeit aus. In den wenigen Fällen (4\%), in denen ein klinisch-krankes EC-infiziertes Tier keine Antikörper hat, geht man davon aus, dass entweder zum Zeitpunkt der Blutentnahme gerade alle Antikörper verbraucht sind oder, ähnlich wie bei Tollwut, dass das Tier keine Antikörper bildet [11].

Eine Testung auf IgM- (akut) und IgGAntikörper wird in Deutschland derzeit noch nicht angeboten. Sie scheint aber auch nicht wirklich hilfreich in Bezug auf die Ursache der klinischen Symptome zu sein, da auch bei $24 \%$ der klinisch gesunden Kaninchen IgM-AK (52\% IgG, n=62) nachgewiesen wurden [13].

\section{Veränderungen bei der Blutuntersuchung}

Diese Veränderungen sind abhängig vom Ausmaß der Organschädigungen sowie von sekundären Infektionen und Problemen. Die E.-cuniculi-Infektion selbst macht zunächst gar keine Blutbildveränderungen (physiologisches lymphozytäres Blutbild). Erst bei länger bestehender Infektion kommt es im Vergleich zu gesunden Tieren signifikant häufiger zu milden, nicht-regenerativen Anämien, bei zunehmender Nierenschädigung zu Harnstoff konzentrationserhöhungen oder bei verstärkten Muskelaktivitäten (Rollen) zu CK-Aktivitätserhöhungen [8]. Beim Vorliegen anderer Blutbildveränderungen sollte nach weiteren Krankheiten/Ursachen gesucht werden.

\section{Differenzialdiagnosen}

Zwei aktuelle deutsche Untersuchungen an Heimtierkaninchen mit „typischen Symptomen“ einer Enzephalitozoonose zeigen, dass nicht wie erwartet $100 \%$ Antikörper gegen E. cuniculi aufweisen, sondern nur 60\% (144/241) [11] bzw. 64\% [4]. Geht man zudem davon aus, dass mindestens die Hälfte der infizierten Kaninchen zumeist symptomfrei ist, bedeutet dies im Umkehrschluss, dass bei bis zu 70\% der verdächtigen Kaninchen eben nicht die Enzephalitozoonose die Ursache der Symptome ist. Die Ausschlussdiagnostik ist also ein sehr wichtiger Anteil der Diagnosestellung [11].

Viele Tiere „gönnen sich“ mehr als nur eine Krankheit: Ein Kaninchen kann durchaus E.-cuniculi-Antikörper aufweisen und trotzdem eine andere Krankheit haben.

Je nach Symptom gibt es eine Vielzahl von Differenzialdiagnosen. Bei neurologischen Symptomen macht man sich gern das „VETAMIN-D-Schema“ zunutzen ( Tab. 1). Zu den häufigsten Differenzialdiagnosen bei Kopfschiefhaltung zählen v. a. die Otitis, das Schädelhirntrauma und die Meningoenzephalitis durch Bakterien und Viren. 
Tab. 1 Häufige Differenzialdiagnosen für Vestibularsyndrom und Parese mit diagnostischem Plan (nach ausführlicher Anamnese und klinischer/neurologischer Untersuchung). In Anlehnung an Flock 2010 [8].

\begin{tabular}{|c|c|c|c|}
\hline VETAMIN D & Vestibularsyndrom (zentral/peripher) & Parese (Para-/Tetraparese) & diagnostischer Plan \\
\hline Vaskulär & $\begin{array}{l}\text { Vaskulitis, Infarkt, kardiovaskuläre } \\
\text { Läsionen }\end{array}$ & $\begin{array}{l}\text { Vaskulitis, Infarkt, kardiovaskuläre } \\
\text { Läsionen }\end{array}$ & - Blutbild \\
\hline Entzündlich & $\begin{array}{l}\text { Enzephalitozoonose, Meningoenze- } \\
\text { phalitis, Otitis (Pasteurella multocida, } \\
\text { Staphylokokken, Escherichia coli, } \\
\text { Pseudomonas, Psoroptes cuniculi, } \\
\text { Herpesvirus, Toxoplasma etc .) }\end{array}$ & $\begin{array}{l}\text { Enzephalitozoonose, bakterielle } \\
\text { Infektionen (Wirbelsäule), andere } \\
\text { Infektionen (Toxoplasmose), } \\
\text { Discospondylitis, Myelopathie etc. }\end{array}$ & $\begin{array}{l}\text { - Differenzialblutbild } \\
\text { - Antikörperbestimmung } \\
\text { - Ohruntersuchung (Röntgen, CT) }\end{array}$ \\
\hline Traumatisch & Schädel-Hirn-Trauma & $\begin{array}{l}\text { Wirbelsäulentrauma, Frakturen, } \\
\text { Luxationen }\end{array}$ & $\begin{array}{l}\text { - CK-Bestimmung } \\
\text { - Röntgen }\end{array}$ \\
\hline Angeboren & $\begin{array}{l}\text { portosystemischer Shunt, Epilepsie (Wei- } \\
\text { ße Wiener) }\end{array}$ & $\begin{array}{l}\text { Splay leg, Schüttellähme (Deutsche } \\
\text { Widder), erbliche Ataxie, spastische } \\
\text { Spinalparalyse, Glykogenspeicher- } \\
\text { krankheit }\end{array}$ & $\begin{array}{l}\text { - Serumprofil } \\
\text { - Sonografie }\end{array}$ \\
\hline Metabolisch & $\begin{array}{l}\text { nutritive Störungen, Hepatoenzephales } \\
\text { Syndrom, Mykotoxine }\end{array}$ & $\begin{array}{l}\text { Toxin (Mykotoxine, Botulismus, etc.), } \\
\text { Diabetes mellitus, Hypokalzämie, } \\
\text { Hypokaliämie etc. }\end{array}$ & $\begin{array}{l}\text { - Blutbild } \\
\text { - Serumprofil }\end{array}$ \\
\hline Idiopathisch & - & - & - Ausschluss anderer Erkrankungen \\
\hline Neoplastisch & Tumoren & Tumoren & $\begin{array}{l}\text { - Differenzialblutbild } \\
\text { - Serumprofil } \\
\text { - Sonografie } \\
\text { - CT, MRT }\end{array}$ \\
\hline Degenerativ & Hitzschlag & Bandscheibenprolaps & $\begin{array}{l}\text { - Blutbild } \\
\text { - Serumprofil }\end{array}$ \\
\hline
\end{tabular}

Ursachen für Paresen sind vor allem Wirbelsäulentraumata und Tumore [8], [10].

Zu einer ausführlichen klinischen Untersuchung gehören bei Verdacht auf Enzephalitozoonose immer [8]:

- Blutuntersuchung mit Differenzialblutbild (Ausschluss anderer Infektionen)

- Muskelenzymaktivitäten (Ausschluss eines Traumas)

- Antikörperbestimmung

\section{Gibt es eine Therapie?}

Laut Literatur sind Antiparasitika wie Fenbendazol und/oder Antibiotika wie Oxytetrazyklin, Chloramphenicol und Gyrasehemmer zur Therapie geeignet [1], [23]. Laut der aktuellsten Studie [21] ist die Überlebensrate bei einer Kombinationstherapie aus Fenbendazol $(20 \mathrm{mg} / \mathrm{kg}$ $1 \times$ tgl. p.o. über 21 Tage) und Antibiose (Oxytetrazyklin $20 \mathrm{mg} / \mathrm{kg} 1 \times$ tgl. s.c. über 10 Tage) besser als bei einer Therapie nur mit Antibiotikum ( $\vee$ Tab. 2).
Tab. 2 Aktuelle Therapieempfehlungen in Anlehnung an Sieg et al. 2012 [21].

\begin{tabular}{|c|c|}
\hline Zielgruppe & Therapie/Prophylaxe \\
\hline \multirow[t]{3}{*}{$\begin{array}{l}\text { Kaninchen mit } \\
\text { klinisch-mani- } \\
\text { fester Enzepha- } \\
\text { litozoonose }\end{array}$} & $\begin{array}{l}\text { Fenbendazol } 20 \text { mg/kg/d p.o. 21(-28) Tage + Antibiotikum (mind. } 10 \\
\text { Tage): } \\
\text { - bei ungestörtem Bewusstsein und Niereninsuffizienz: z. B. Enrofloxa- } \\
\text { cin ( } 10 \mathrm{mg} / \mathrm{kg} \text { p.o.) } \\
\text { bei gestörtem Bewusstsein und Sekundärinfektion: sicher ZNS- } \\
\text { gängiges Antibiotikum wie Oxytetracyclin ( } 20 \mathrm{mg} / \mathrm{kg} / \mathrm{d} \text { s.c.) oder } \\
\text { Chloramphenicol ( } 50 \mathrm{mg} / \mathrm{kg} 2 \times \text { tgl. p.o.) }\end{array}$ \\
\hline & $\begin{array}{l}\text { zusätzlich je nach Allgemeinbefinden: } \\
\text { - Aufrechterhaltung der Futteraufnahme (ggf. Zwangsfütterung) } \\
\text { - Infusionen (mind. Erhalt [50 ml/kg/d] + Defizit + Verlust s.c. oder i.v.) } \\
\text { - Vitamin B (je nach Präparat) } \\
\text { - Augensalbe (mit Tetrazyklinen) } \\
\text { - Anfällen: ggf. kurzzeitig Diazepam (0,1 mg/kg s.c.) }\end{array}$ \\
\hline & $\begin{array}{l}\text { Therapiebeginn bei Verdacht sofort bei Erstvorstellung mit Anpassung } \\
\text { nach Vorliegen der Befunde! }\end{array}$ \\
\hline $\begin{array}{l}\text { E.-cuniculi-posi- } \\
\text { tive Tiere } \\
\text { (+ Partnertiere) }\end{array}$ & $\begin{array}{l}\text { Klinisch inapparente Tiere (nach 1. Schub) u./o. Begleittiere bei } \\
\text { vorhersehbarer Immunsuppression (Krankheit, Partnerwechsel, } \\
\text { Tierarztbesuch etc.): } \\
\text { - Fenbendazol ( } 20 \mathrm{mg} / \mathrm{kg} / \mathrm{d} \text { p.o., 3-10 Tage je nach Häufigkeit und } \\
\text { Dauer der Immunsuppression) }\end{array}$ \\
\hline $\begin{array}{l}\text { E.-cuniculi-nega- } \\
\text { tive Tiere }\end{array}$ & $\begin{array}{l}\text { Testung zur Schaffung von E.-cuniculi-freien Beständen, Aufklärung, } \\
\text { Hygiene }\end{array}$ \\
\hline Besitzer & $\begin{array}{l}\text { - Hygiene im Umgang mit dem Urin positiver Kaninchen } \\
\text { Kontakt zwischen positiven Kaninchen und immunsupprimierten } \\
\text { Personen (HIV, Chemotherapie etc.) vermeiden }\end{array}$ \\
\hline
\end{tabular}


.konkret

Die lange Zeit verwendete Gabe von Glukokortikoiden über einen oder mehrere Tage gilt heute als obsolet.

Glukokortikoide führten in keiner der Kombinationen (Antibiose \pm Fenbendazol) zu einer Verbesserung [21], bergen viele Nebenwirkungen (Lebertoxizität bei steroidsensiblen Kaninchen, Immunsuppression, Wundheilungsstörung, Hyperglykämie etc.) und sind bei Tieren mit zusätzlichen Infektionen durch die entstehende Immunsuppression oft fatal [18].

Unterstützend zur Therapie können Vitamin B und ggf. eine tetrazyklinhaltige Augensalbe gegeben werden. Die weitere Therapie erfolgt symptomatisch. Futteraufnahme und Hydratationsstatus müssen sicher gestellt sein.

Die Therapie mit Fenbendazol und Antibiose sollte bei Verdacht auf Enzephalitozoonose sofort bei der Erstvorstellung beginnen und dann nach Vorliegen der Blutbefunde entsprechend angepasst werden.

Nach Erfahrungen der Autorin sollten Begleittiere (vermeintlich klinisch inapparente Träger) ebenfalls über einige Tage (ca. 5-10, je nach Dauer der Immunsuppression) mit Fenbendazol behandelt werden, um eine klinische Manifestation zu verhindern. Die dauerhafte oder intervallweise Gabe von Fenbendazol scheint bei immunkompetenten Tieren nicht erforderlich, Studien hierzu fehlen noch.

\section{Prognose}

Die Prognose bei klinisch manifester Enzephalitozoonose ist abhängig von der Nierenfunktion [8]. Nach Therapiebeginn ist bis zum Erreichen des Wirkspiegels noch eine weitere Erstverschlechterung über 1-2 Tage möglich. Ab Tag 3 sollte jedoch - abhängig von den sonstigen Befunden - eine erkennbare Besserung eintreten. Paresen und andere neurologische Symptome (auch starkes Rollen) verschwinden bei frühzeitigem Therapiebeginn meist innerhalb von 10 Tagen, eine leichte Kopfschiefhaltung kann ggf. zurückbleiben [22].

\section{Ist eine Erregerelimination möglich?}

Eine Erregerelimination ist bisher nicht möglich. Auch das Vorhandensein von Antikörpern schützt nicht gegen Rezidive, da die Immunität v.a. von der T-ZellFunktion abhängig ist [25]. Nach Erfahrung der Autorin können Rezidive jedoch zumeist vermieden werden durch stressarme Haltung, Unterstützung des Immunsystems und medikamentelle Reduktion zirkulierender Erreger in Stressphasen ( $\triangleright$ Tab.2).

\section{Schutz von Menschen und Partnertieren}

Menschen erkranken nur sehr selten und auch nur dann, wenn ihr Immunsystem durch eine andere schwere Krankheit (HIV, Chemotherapie etc.) stark geschwächt ist und sie große Erregermengen aufnehmen [5]. Diese Menschen sollten den Kontakt zu Antikörper-positiven Tieren und v.a. ihrem Urin meiden. Bei gesunden Personen sollten der vorsichtige Umgang mit dem Urin der Tiere und das Händewaschen nach Kontakt ausreichend sein.

Am gefährdetsten sind die Partnertiere (auch Meerschweinchen), da sie ständig Kontakt zu den infizierten Kaninchen und ihrem Urin haben. Ein wirksamer Schutz vor Infektion ist bei ihnen nicht möglich. Antikörper-negative Tiere sollten daher nicht mit Antikörper-positiven vergesellschaftet werden. Sind die Tiere bereits zusammen, gelten die Partnertiere automatisch auch als „E.-cuniculi-Träger“.

\section{Fazit für die Praxis}

Die Enzephalitozoonose ist eine Zoonose. Oberstes Ziel muss es daher sein, die Trägertiere zu identifizieren. Nur so kann die weitere Verbreitung der Infektion dauerhaft reduziert werden und infizierte Tiere können optimal therapiert und vor Rezidiven geschützt werden. Essentiell für eine optimale Therapie sind die Antikörperbestimmung und die Ausschlussdiagnostik, denn bei bis zu 70\% der verdächtigen Kaninchen ist die Enzephalitozoonose nicht die Ursache der Symptome.
Online zu finden unter

http://dx.doi.org/10.1055/s-0035-1558503

\section{Literatur}

1 Beauvais B, Sarfati C, Challier S et al. In vitro model to assess effect of antimicrobial agents on Encephalitozoon cuniculi. Antimicrob Agent Chemother 1994; 38 (10): 2440-2448

2 Botha WS, van Dellen AF, Stewart CG. Canine encephalitozoonosis in South Africa. J $S$ Afr Vet Assoc 1979; 50 (2): 135-144

3 Cox JC, Hamilton RC, Attwood HD. An investigation of the route and progression of encephalitozoon cuniculi infection in adult rabbits. J Protozool 1979; 26 (2): 260-265

4 Csokai J, Weider K, Müller E. Prevalence of Encephaitozoon cuniculi infections in Germany. Poster FECAVA München 2014

5 Deplazes P, Mathis A, Baumgartner R et al. Immunologic and molecular characteristics of Enzephalitozoon-like microsporidia isolated from humans and rabbits indicate that Encephalitozoon cuniculi is a zoonotic parasite. Clin Infect Dis 1996; 22 (3): 557-559

6 Ewringmann A, Göbel T. Untersuchungen zur Klinik und Therapie der Enzephalitozoonose beim Heimtierkaninchen. Kleintierprax 1999; 44: 357-372

7 Flatt RE, Jackson SJ. Renal nosematosis in young rabbits. Pathol Vet 1970; 7 (6): 492-497

8 Flock U. Enzephalitozoonose beim Kaninchen - eine retrospektive Auswertung [Dissertation]. München: LMU München; 2010

9 Gannon J. A survey of encephalitozoon cuniculi in laboratory animal colonies in the united kingdom. Lab Anim 1980; 14 (2): 91-94

10 Harcourt-Brown FM, Holloway HK. Encephalitozoon cuniculi in pet rabbits. Vet Rec 2003; 152: 427-431

11 Hein J, Flock U, Sauter-Louis C et al. Encephalitozoon cuniculi in rabbits in Germany: prevalence and sensitivity of antibody testing. Vet Rec 2014; 174 (14): 350, DOI: 10.1136/vr.102126

12 Jass A, Matiasek K, Henke J et al. Analysis of cerebrospinal fluid in healthy rabbits and rabbits with clinically suspected encephalitozoonosis. Vet Rec 2008; 162 (19): 618-622

13 Jeklova E, Jekl V, Kovarcik K et al. Usefulness of detection of specific $\mathrm{lgM}$ and $\mathrm{lgG}$ antibodies for diagnosis of clinical encephalitozoonosis in pet rabbits. Vet Parasitol 2010; 170 (1-2): 143-148, DOI: 10.1016/j.vetpar. 2010.01.029. 
14 Künzel F, Joachim A. Encephalitozoonosis in rabbits. Parasitol Res 2010; 106 (2): 345-349

15 Mathis A. Microsporidia: Emerging advances in understanding the basic biology of these unique organisms. Int J Parasitol 2000; 30 (7): 795-804

16 Meyer-Breckwoldt A. Epidemiologische und klinische Untersuchungen zur Enzephalitozoonose beim Zwergkaninchen [Dissertation]. Hannover: Stiftung Tierärztliche Hochschule Hannover; 1996

17 Neuwirth E. Enzephalitozoonose [Dissertation]. Hannover: Stiftung Tierärztliche Hochschule Hannover; 1988

18 Rosenthal KL. Therapeutic Contraindications in Exotic Pets. J Exot Pet Med 2004; 13 (1): 44-48

19 Scharmann W, Reblin L, Griem W. Infection of rabbits with encephalitozoon cuniculi. Berl Munch Tierarztl Wochenschr 1986; 99 (1): 20-24
20 Shadduck JA, Watson WT, Pakes SP et al. Animal infectivity of encephalitozoon cuniculi. J Parasitol 1979; 65 (1): 123-129

21 Sieg J, Hein J, Jass A et al. Clinical evaluation of therapeutic success in rabbits with suspected encephalitozoonosis. Vet Parasitol 2012; 187 (1-2): 328-332

22 Sieg J. Enzephalitozoonose beim Kaninchen - eine Therapiestudie [Dissertation]. München: LMU München; 2014

23 Suter C, Muller-Doblies UU, Deplazes P et al. Prevention and treatment of encephalitozoon cuniculi infection in rabbits with fenbendazole. Vet Rec 2001; 148: 478-480

24 Valencakova A, Revajova V, Balent P et al. Immunosuppressive effect of Encephalitozoon cuniculi. Bull Vet Inst Pulawy 2003; 47: $113-120$

25 Valencakova A, Halanova M. Immune response to Encephalitozoon: review. Comp Immunol Microbiol Infect Dis 2012; 35 (1): $1-7$
26 Varga M. Questions around Encephalitozoon cuniculi in rabbits. Vet Rec 2014; 174 :

347-348

27 Waller T, Morein B, Fabiansson E. Humoral immune response to infection with encephalitozoon cuniculi in rabbits. Lab Anim 1978; 12 (3): 145-148

28 Wright JH, Craighead EM. Infectious motor paralysis in young rabbits. J Exp Med 1922; 36 (1): 135-140

\section{Dr. Jutta Hein}

Diplomate ECZM (Small Mammal)

Zbz. Heimtiere/Kleinsäuger

Kleintierpraxis am Klinkerberg, Augsburg

Synlab.vet Augsburg

dr.hein@heimtiertieraerztin.de

www.heimtieraerztin.de 